\title{
ERPs reveal similar effects of social gaze orienting and voluntary attention, and distinguish each from reflexive attention
}

\author{
Vicki W. Chanon • Joseph B. Hopfinger
}

Published online: 20 September 2011

(C) Psychonomic Society, Inc. 2011

\begin{abstract}
Spatial attention can be reflexively captured by a physically salient stimulus, effortfully directed toward a relevant location, or involuntarily oriented in the direction of another person's gaze (i.e., social gaze orienting). Here, we used event-related potentials to compare the effects of these three types of orienting on multiple stages of subsequent target processing. Although gaze orienting has been associated more strongly with reflexive capture than with voluntary attention, the present data provide new evidence that the neural effects of social gaze orienting are markedly different from the effects of reflexive attentional capture by physically salient stimuli. Specifically, despite their similar behavioral effects, social gaze orienting and reflexive capture produce different effects on both early sensory processing ( $\sim 20 \mathrm{~ms}$; $\mathrm{P} 1 / \mathrm{N} 1$ components) and later, higher-order processing ( $\sim 300 \mathrm{~ms} ;$ P3 component). In contrast, the effects of social gaze orienting were highly similar to those of voluntary orienting at these stages of target processing.
\end{abstract}

Keywords Endogenous · Exogenous · Attention · Joint attention Event-related potential

An enormous amount of information is presented to our sensory systems at any given moment. The ability to efficiently focus on a subset of this otherwise overwhelmingly complex environment depends on mechanisms of

\footnotetext{
V. W. Chanon · J. B. Hopfinger $(\bowtie)$

Department of Psychology,

University of North Carolina at Chapel Hill,

CB 3270, Davie Hall,

Chapel Hill, NC 27599-3270, USA

e-mail: hopfinger@unc.edu

V. W. Chanon

e-mail: vmwest@email.unc.edu
}

attention. Previous research has largely focused on two modes of orienting attention: a rapid and automatic reflexive capture of attention by salient stimuli, and a slower and more effortful voluntary orienting (e.g., Cheal \& Lyon, 1991; Desimone \& Duncan, 1995; Jonides, 1981; Müller \& Rabbitt, 1989; Posner, 1980). More recently, however, attention has been shown to be under the influence of social cues as well. Social gaze orienting refers to the situation in which the location being gazed at by another person affects the attentional allocation of the observer. Specifically, the observer's responses to targets are faster and more accurate when targets appear at the gazed-at location versus at other locations (e.g., Driver, Davis, Ricciardelli, Kidd, Maxwell and Baron-Cohen 1999; Friesen \& Kingstone, 1998). It is unclear, however, where social gaze orienting fits into the classic dissociation between voluntary and involuntary attention.

Although it is generally agreed that attention can be oriented in different manners, there is uncertainty regarding whether different types of orienting simply reflect different modes of controlling a unitary focus of attention, or rather whether different types of orienting rely on distinct systems that bias subsequent neural processing in unique ways. Evidence has suggested that voluntary orienting and reflexive capture may represent at least partially separable systems with somewhat different effects on responding to an attended target (Berger, Henik, \& Rafal, 2005; Briand, 1998; Prinzmetal, McCool, \& Park, 2005). In addition, recent work testing various models of attention has suggested that voluntary and involuntary attention may affect behavioral responses through different means. For example, $\mathrm{Lu}$ and Dosher (2000) concluded that an informative central precue (measuring voluntary attention) enhances stimulus processing to a target location primarily by reducing external noise, whereas a peripheral onset 
(measuring involuntary attention) not only reduces external noise, but also enhances perceptual processing of the signal. Prinzmetal et al. (2005) also concluded that voluntary attention can enhance the perceptual representation of the target, but later work (Prinzmetal, Ha, \& Khani, 2010) led to the conclusion that reflexive attention works at a decision stage of processing. Specifically, independent decisions are made for targets at each possible location until enough evidence accrues and a response threshold is reached for one target. Although we did not directly test these models of attention in the present study, there is growing evidence for the distinction between voluntary and involuntary attention and the different effects that each has on target processing. The main question here was whether social gaze orienting works via neural effects that are similar to either voluntary or reflexive attention, or whether social gaze orienting represents a third distinct attention system.

Social gaze orienting is sometimes characterized as being similar to reflexive attention and different from voluntary orienting. For example, reflexive and social gaze orienting, unlike voluntary attention, are not dependant on the cue being predictive of the target location. Indeed, social gaze orienting effects have been found even when the gaze was known to be counterpredictive of target location (e.g., Bayliss \& Tipper, 2006; Driver et al. 1999; Friesen \& Kingstone, 1998). Furthermore, in a study that used counterpredictive gaze cues with multiple potential target locations, responses to targets were enhanced both at the location of the directed gaze and at the predicted location (i.e., opposite the gaze) relative to other locations, suggesting that voluntary and social attention may be distinct (Friesen, Ristic, \& Kingstone, 2004). Such findings have led to suggestions that social gaze attention may be more similar to reflexive than to voluntary attention.

Other studies, however, have provided evidence that the effects of social gaze orienting also differ from reflexive attention effects in some respects. For example, Friesen and Kingstone (2003) found that gaze cuing speeds reaction times, not only at short cue-to-target intervals, but also at long cue-to-target intervals, at which reflexive attention typically produces a slowing of reaction times, referred to as inhibition of return (IOR; Posner \& Cohen, 1984). Therefore, based on behavioral measures of target responding, it has remained unclear to what extent social gaze orienting exerts its effects in a manner separate from, versus shared with, voluntary or reflexive attention.

Although behavioral differences have been found between gaze orienting, voluntary attention, and reflexive capture, overt responses can obscure effects on neural processing that precede the behavioral responses. Differences in behavioral measures may mask similar effects at early stages of processing, and conversely, differences at earlier stages of processing may not be observable in behavioral measures. Previous studies have shown the utility of using the event- related potential (ERP) technique to identify effects on early stages of processing that are sometimes obscured in behavioral performance (e.g., Handy, Green, Klein, \& Mangun, 2001). Investigating the neural effects that precede overt responses can be critical for better understanding the multiple influences and operations underlying the dynamic allocation of attentional resources.

In the present study, we used ERPs to compare the effects of voluntary, reflexive, and social orienting on multiple stages of target processing. We focused on two components of target processing that have previously been shown, across many studies, to be sensitive to attention. Furthermore, these two components index distinct stages of processing, and therefore relate to theories that posit that attention works at perceptual versus decision-level stages. Specifically, we investigated sensory-level processing, as indexed by the P1 component ( $100-\mathrm{ms}$ latency), and higher-order semantic/decision-level processing, as indexed by the P3 ( 300-ms latency).

Recent results have provided strong evidence that modulation of early sensory-evoked components (e.g., P1) following an involuntary shift of attention is strongly linked to enhanced perceptual ability (Störmer, McDonald, \& Hillyard, 2009). Modulation of later stages of processing (e.g., P3) would suggest effects that are on postperceptual levels, such as may be predicted by models positing that attention works on decision-level stages. Previous studies of involuntary attention have consistently found, across different tasks, that reflexive attention enhances both the $\mathrm{P} 1$ and the $\mathrm{P} 3$. The effects of voluntary attention have been more variable; while many studies have shown P1 and P3 effects, task and display parameters affected the presence of these effects.

In previous studies researchers have used ERPs to investigate social gaze orienting, but we know of no research that has directly compared social gaze orienting to both voluntary and involuntary orienting. Furthermore, the existing ERP studies of social gaze cuing have not produced consistent results. For example, some studies have found that gaze cuing enhances the amplitude of the target-evoked P1 component (e.g., Schuller \& Rossion, 2001, 2004), while others have shown no evidence of amplitude modulation of early visual processing by social gaze cuing (Fichtenholtz, Hopfinger, Graham, Detwiler, \& LaBar, 2007, 2009; Magnée, Kahn, Cahn, \& Kemner, 2011). Thus, it may be the case that the ERP effects of social gaze orienting may be sensitive to the perceptual and cognitive loads induced by the task and stimuli, as has been shown for voluntary attention (e.g., Eimer, 1994a; Handy \& Mangun, 2000; Prime \& Ward, 2006; Vogel \& Luck, 2000). In addition, theories of attention based on overt behavior have often assumed multiple types of attention processes, and that some mechanisms may be differentially involved, depending on the task and stimulus parameters (see, e.g., 
Lu \& Dosher, 1998, and Prinzmetal et al. 2005). This highlights the importance of within-subjects comparisons using the same task and stimuli when comparing attention effects across types of orienting.

Two recent studies have used a within-subjects design to compare the ERP effects of gaze orienting to other types of orienting, but the results have been inconsistent. Tipper, Handy, Giesbrecht, and Kingstone (2008) used a novel, static cue stimulus that could be interpreted as either an arrow pointing in one direction or an eye looking in the opposite direction. The results showed a P1 amplitude effect for targets following the cue when it was interpreted as an eye, but not when the stimulus was supposed to be viewed as an arrow. Although that study provided evidence that adding a social component to a nonpredictive cue stimulus may alter the effects of that stimulus, it did not directly compare social gaze orienting to voluntary orienting (in that it did not include a condition with a predictive cue stimulus). Furthermore, the stimulus in that study was unique, different from the arrow and social gaze cues used in all previous studies. Hietanen, Leppänen, Nummenmaa, and Astikainen (2008) used a more typical gaze cuing stimulus (two eyes, with the pupils being directed in one direction or the other) and a more standard arrow cue stimulus, and they found no cuing/validity effect on the P1 amplitude following either gaze or arrow cues. As in the Tipper et al. study, the arrow cue was nonpredictive of target location, so neither study had a condition that isolated either purely voluntary attention or sensory-induced involuntary capture.

Although the effect of social gaze cuing on the $\mathrm{P} 1$ has been variable across studies, the effects on the $\mathrm{P} 3$ component have been more consistent, albeit less consistently reported. Specifically, invalid targets have consistently produced a larger-amplitude P3 than valid targets following a social gaze cue, even when both locations were equally probable and equally task-relevant (Fichtenholtz, Hopfinger, Graham, Detwiler and LaBar 2007, 2009; Magnée et al. 2011; Schuller \& Roisson, 2001, 2005). We know of no studies that have found the opposite pattern (with valid targets producing a stronger $\mathrm{P} 3$ than invalid targets).

The P3 component (occurring at $\sim 300-800 \mathrm{~ms}$ ) has been found to be related to the perceived relevance of a stimulus and to context updating (Donchin, 1981; Duncan-Johnson \& Donchin, 1982). Since the effect on the P3 has consistently been found across studies that have reported P3 analyses, it may play an important role in the consistent behavioral facilitation seen across studies. Therefore, the P3 may be a more robust measure with which to observe any differences between these types of attentional orienting than are differences in earlier perceptual processing, which may be less strongly linked to the behavioral effects of social gaze orienting. We discuss this effect of social gaze orienting more fully in the Discussion section.
In the present study, we examined ERPs to targets following three distinct cue types (voluntary, reflexive, and social gaze cues) to gain a better understanding of which operations are similar and different across these types of orienting. Since we are interested in the differential effects on processing targets following each type of orienting, the target events were identical across conditions, whereas the cue events were different, because these were designed to isolate each type of attention. By comparing the stages of processing at which each of these cues exerted its effects, we hoped not only to gain a better understanding of how social gaze orienting compares to voluntary and reflexive attention, but to gain additional knowledge about the operations involved in all three types of attention.

\section{Method}

Participants

The participants were students at the University of North Carolina at Chapel Hill, who were recruited via flyers and mass e-mail letters. Participants received $\$ 10 / \mathrm{h}$, and the experiment lasted approximately $3 \mathrm{~h}$. One of the participants was excluded due to excessive blinking. The final analysis included data from 16 participants ( 7 female) with a mean age of 22.8 years (range $=18-29)$.

\section{Materials and procedure}

The experiment consisted of three different types of blocks: (1) social gaze orienting, (2)reflexive orienting, and (3) voluntary orienting. A 3-D-rendered computer-generated male figure (presented from the shoulders up and constructed using POSER software, Version 6; e-frontier/ Curious Labs, Scotts Valley, CA) was presented at the center of the screen, with 3-D-rendered cylinders to the left and to the right of the face. This computer-generated figure was present on the screen at all times, controlling for the presence of this stimulus across all conditions. A fixation cross appeared between the eyes of the figure. Participants performed a localization task requiring them to respond to the location of a target (a blue 3-D-rendered ball). The target was presented for $100 \mathrm{~ms}$ to the left or right of the face, directly above one of the cylinders. We attempted to make the presentation of the target stimulus more realistic than in many previous studies. Specifically, as opposed to a target stimulus suddenly appearing and disappearing from empty space, the target stimulus in this experiment could be viewed as a ball briefly popping up out of a cylindrical tube. The diameter of the target ball was $3.68^{\circ}(4.5 \mathrm{~cm}$ at a viewing distance of $70 \mathrm{~cm}$ ), with the center falling $12.10^{\circ}$ to the left or right of the fixation cross on the same plane. For 
all three types of blocks, participants were required to respond with their right hand, pressing one button with their index finger if the target appeared on the left, and another button with their middle finger if it appeared on the right.

To ensure that we would be able to compare ERPs from conditions that all produced robust behavioral effects, we first performed a pilot behavioral study in which there were three different cue-to-target SOA ranges (50-250, 300-500, or 600-800 ms). In the present ERP study, we used the SOA for each type of cuing that produced the strongest effect on reaction times, because we wanted to investigate whether similar neural effects underlie behavioral facilitation across these types of orienting. Our pilot study revealed that the maximal behavioral cuing effects for the reflexive and social gaze orienting conditions occurred at the 50- to 250-ms SOA, whereas the maximal cuing effect in the voluntary orienting condition occurred at the 600 - to $800-\mathrm{ms}$ SOA. These findings are consistent with previous studies investigating each of these types of attention separately, and these are the SOAs used in the present ERP study (50-250 ms for reflexive and social orienting; 600-800 ms for voluntary orienting).

For the social gaze cue blocks, the "cue" event was the eyes of the face moving toward either the left or right peripheral location. The eyes returned to looking straight ahead $600 \mathrm{~ms}$ after the onset of the target, which was followed by a 1,650 - to $2,150-\mathrm{ms}$ intertrial interval (ITI). Eye gaze direction was nonpredictive of where the target would occur. In both the reflexive and voluntary orienting blocks, the face in the center of the screen kept its gaze directed straight ahead through the entire block. The reflexive "cue" event consisted of a white ring (diameter of ring $=4.49^{\circ}$ of visual angle) appearing for $34 \mathrm{~ms}$ in the location of one of the two possible target locations, and again the cue was not predictive of target location. The target event was followed by a 2,250- to 2,750-ms ITI. The voluntary "cue" event was a change in the color of the fixation cross, which remained until the target appeared. The fixation cross changed to red to indicate that the target was likely to appear in the right visual field, or green (isoluminant with the red fixation) to indicate that the target was likely to appear in the left visual field. In these blocks, unlike in the social and reflexive blocks, the cue was $75 \%$ predictive, giving the participants incentive to direct attention to the valid side. A 1,700- to 2,200-ms ITI followed the target event in each trial. (See Fig. 1 for a schematic of a trial and for examples of the different cues.)

\section{Recording and analysis}

Behavioral measures (accuracy and reaction times to the targets) were collected in addition to recording the electroencephalogram (EEG) from 96 electrode sites. The EEG data were referenced to the right mastoid, amplified at a bandpass of $0.05-100 \mathrm{~Hz}$, and digitized at 250 samples per second. Eye movements were monitored online throughout all blocks with the use of a zoom-lens camera. Additionally, electrodes located beneath and lateral to the outer canthus of each eye recorded the electrooculogram. All trials containing eye movements or blinks were rejected offline and not included in the analysis. EEG data were averaged offline to create ERP waveforms. The data were low-pass filtered (24.06-Hz cutoff) to remove high-frequency noise, as well as high-pass filtered with a single-pole causal filter $(0.51 \mathrm{~Hz})$ to reduce low-frequency drifts.

Because the cues and targets in the social gaze and reflexive attention blocks occurred temporally close to one another, the ADJAR (ADJAcent Response estimation and removal) technique was used to remove overlapping activity by convolving the cue and target waveforms with previous and subsequent event distributions (Woldorff, 1993). The ADJAR filter was applied to the individual participant averages before participants' data were averaged together, as in previous studies (Hopfinger \& Mangun, 1998, 2001; Hopfinger \& Ries, 2005; Hopfinger \& West, 2006; McDonald \& Ward 2000). Ten iterations of the ADJAR procedure were performed, and waveforms typically stabilized after 3-6 iterations, suggesting that the procedure was robust and effective. All statistics and plotted waveforms below represent the visual field contralateral to the target location, with the exception of components for which only midline electrodes were tested (N2 and P3). Topographic maps were created by collapsing across visual fields and plotting ipsilateral electrodes on the left side of the head and contralateral electrodes on the right.

\section{Results}

\section{Behavioral results}

For reaction time measures, a $3 \times 2 \times 2$ ANOVA with the factors attention type, visual field, and validity revealed a significant main effect of validity [valid $=319.9 \mathrm{~ms}$, invalid= $373.9 \mathrm{~ms} ; F(1,15)=50.70, p<.001]$, as well as a significant interaction between attention type and validity $[F(2,14)=$ $5.28, p=.011]$. Separate $2 \times 2$ ANOVAs with the factors visual field and validity were conducted for each type of attention (see Table 1 for all of the means and standard deviations). Despite the cuing effect being numerically larger in the voluntary condition than in the others, these separate ANOVAs revealed a significant and robust cuing effect for each type of orienting [voluntary cues, $F(1,15)=18.4, p<.001$; reflexive cues, $F(1,15)=10.0$, $p=.006$; social cues, $F(1,15)=23.2, p<.001]$.

For accuracy measures, a $3 \times 2 \times 2$ ANOVA with the factors attention type, visual field, and validity revealed a 
Fig. 1 An example of a trial with the social gaze cue. Examples of the voluntary and reflexive cues are shown at the bottom of the figure

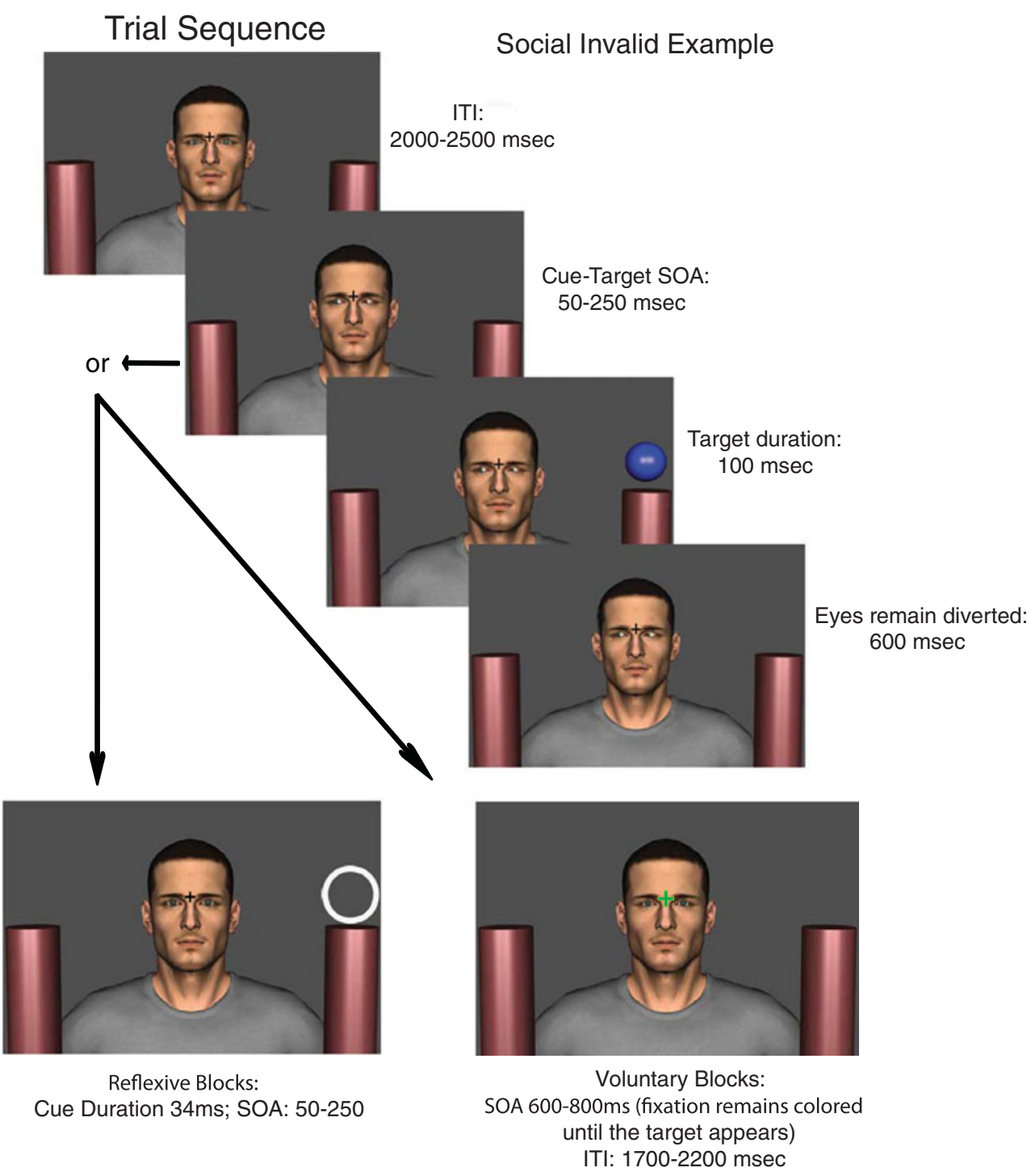

significant main effect of validity [valid $=99.1 \%$, invalid $=$ $96.8 \% ; F(1,15)=16.8, p=.001]$ and no interaction between attention type and validity $[F(2,14)=1.153, p=.34]$. Additional ANOVAs for each type of attention separately

Table 1 Behavioral Results

\begin{tabular}{lll}
\hline Condition & Mean Reaction Time $(S D)$ & Mean Accuracy $(S D)$ \\
\hline Voluntary & & \\
Valid & $269.3(59.1)$ & $99.0(1.3)$ \\
Invalid & $399.5(99.8)$ & $96.3(4.6)$ \\
Reflexive & & \\
Valid & $330.9(64.9)$ & $99.1(1.1)$ \\
Invalid & $364.4(67.5)$ & $96.1(4.2)$ \\
Social Gaze & & $99.2(0.8)$ \\
Valid & $320.2(67.8)$ & $97.2(2.7)$ \\
Invalid & $361.0(72.2)$ & \\
\hline
\end{tabular}

(see Table 1 for the means and standard deviations) confirmed significant effects of validity for voluntary attention $[F(1,15)=10.6, p=.005]$, reflexive attention $[F(1,15)=10.8, p=.005]$, and social attention $[F(1,15)=$ $10.4, p=.006]$. Together, these reaction time and accuracy results show that the choice of SOAs was effective in producing significant behavioral facilitation for each type of orienting. The purpose of the present study was to better understand whether this common behavioral outcome (faster and more accurate responses at the valid location) across all three types of attention is associated with similar effects at each stage of neural processing.

\section{ERP results}

Early visual processing effects The earliest attention effects were observed at posterior scalp sites contralateral to the 
target stimulus. Critically, this effect occurred only for reflexive orienting, with cued-location targets evoking enhanced processing relative to uncued-location targets (Fig. 2). Social and voluntary orienting showed no effects at this stage of processing. This validity effect for the reflexive orienting condition peaked at a $127-\mathrm{ms}$ latency, as indicated by the difference wave (valid minus invalid targets; Fig. 2b). The timing of this effect lies between the peaks of the P1 and N1 components, similar to previous findings of involuntary orienting to salient visual stimuli (Hopfinger \& Ries, 2005; Hopfinger \& West, 2006). We conducted a $3 \times 2 \times 2 \times 2$ ANOVA with the factors attention type (voluntary, reflexive, and social), validity (valid and invalid), visual field (right visual field [RVF] and left visual field $[\mathrm{LVF}]$ ), and electrode (two electrode sites circled in Fig. 2c), to test for differences in the mean amplitude within the latency range of $117-137 \mathrm{~ms}$. This ANOVA revealed no main effect of validity $[F(1,15)=2.67$, $p=.12]$; however, there was a significant interaction between attention type and validity $[F(2,14)=9.08, p<.001]$.
To probe the critical finding of a significant attention type $\times$ validity interaction, separate ANOVAs were conducted with the same factors as above, but with just two of the attention types at a time (i.e., voluntary vs. reflexive, voluntary vs. social, and reflexive vs. social). There were significant interactions of attention type and validity for both the reflexive and voluntary attention comparison $[F(1,15)=172.53, p<.001]$ and the reflexive and social attention comparison $[F(1,15)=7.61, p<.05]$. These interactions provided evidence that reflexive orienting produces an effect that is not produced for social and voluntary orienting. In the analysis comparing voluntary and social attention, the interaction between attention type and validity was not significant $[F(1,15)=0.33, p=.57]$.

Finally, a separate $2 \times 2 \times 2$ ANOVA with the factors validity, visual field, and electrode was conducted for each type of attention. There was no significant main effect of validity, nor any interactions with validity, for social gaze orienting [validity: $F(1,15)=0.01, p=.91$ ] or voluntary orienting [validity: $F(1,15)=1.56, p=.23$ ]. For reflexive

\section{a Target ERPs (Early occipital components)}
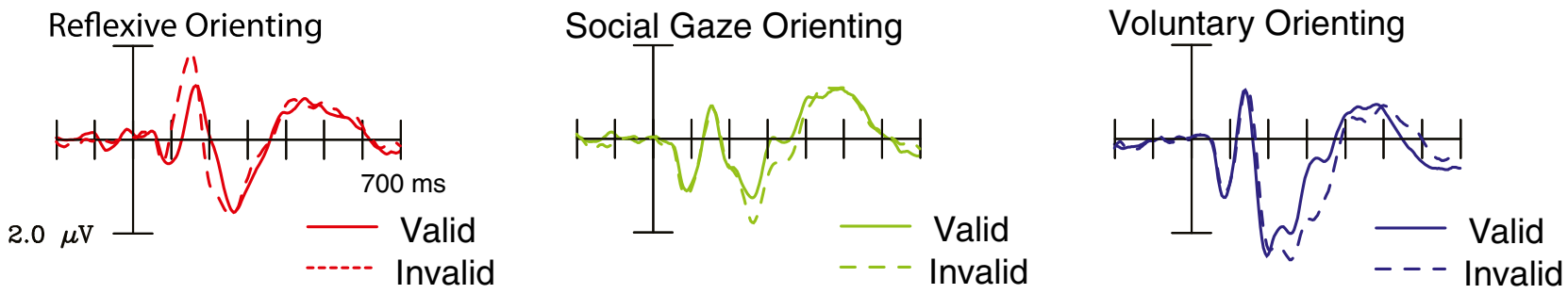

\section{b}

Difference waves (valid -invalid)

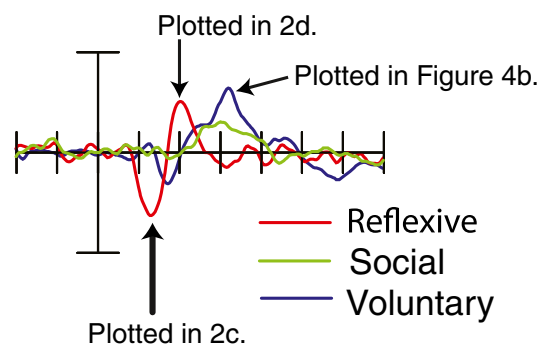

C Map of reflexive attention effect (valid - invalid) from 117-137ms d Map of reflexive attention effect (valid - invalid) from 190-210 ms

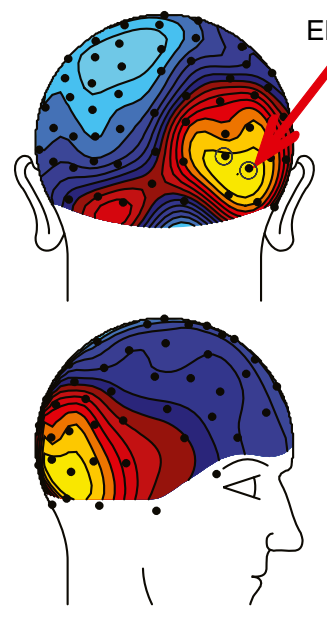

Electrode plotted $2 \mathrm{a}$ and $2 \mathrm{~b}$.
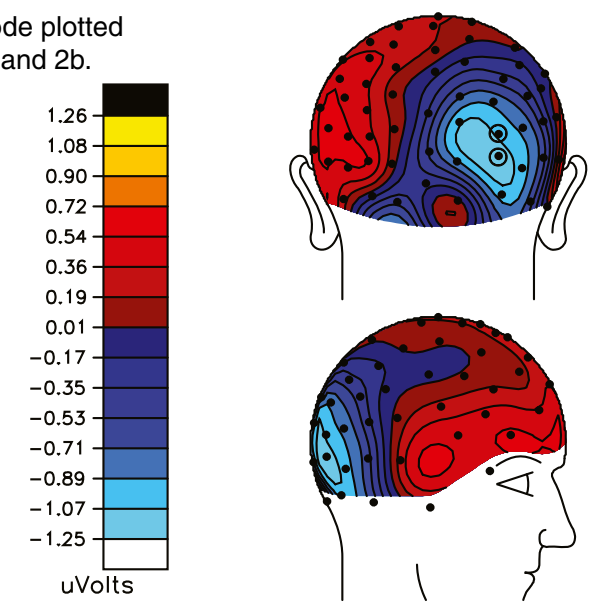

Fig. 2 Early occipital components. (a) Plots of valid and invalid waveforms for each type of attention, plotted at the electrode locations indicated in 2c. (b) Valid-minus-invalid difference waveforms for each attention type, at the electrodes indicated in 2c. Reflexive attention is the only type showing validity differences at this early processing stage. (c) Topographic map of the reflexive attention effect (indicated on the difference wave plotted in $2 b$ ), for the time range of 117 $137 \mathrm{~ms}$. The two electrodes that are circled were the ones used in the statistical analyses. (d) Topographic map of the reflexive attention effect (indicated on the difference wave plotted in $2 b$ ), for the time range of 190-210 ms. The two electrodes that are circled were the ones used in the statistical analyses 
blocks, however, there was a significant main effect of validity $[F(1,15)=8.29, p<.05]$. Thus, although all three conditions produced significant cuing effects in behavioral measures, early sensory processing was only modulated by reflexive orienting, suggesting that social and voluntary attention produced behavioral effects in the present task via different neural operations.

Although it was not of a priori interest, another difference between reflexive attention and the other two types of attention was observed at similar posterior sites at the $\sim 200$ ms latency. Specifically, the present data revealed a negativity for valid versus invalid targets that was observed only in the reflexive attention condition (Fig. 2a). The timing and topography of this component (Fig. 2d) suggest that the difference may be similar to the "negative difference" $(\mathrm{Nd})$ component reported previously in studies of crossmodal attention (i.e., Kennett, Eimer, Spence, \& Driver, 2001; McDonald, Teder-Sälejärvi, DiRusso, \& Hillyard, 2003; McDonald, Teder-Sälejärvi, Heraldez, \& Hillyard, 2001). There was no effect in this latency range for social and voluntary attention (Fig. 2a). An ANOVA over the latency range of 190-210 ms with the factors listed above (but for slightly more medial electrodes; electrode sites circled in Fig. 2d) revealed a significant interaction between attention type and validity $[F(2,14)=9.01, p<.001]$. Separate followup ANOVAs for the different types of attention showed a significant validity difference for reflexive attention [valid = $1.23 \mu \mathrm{V}$, invalid $=0.13 \mu \mathrm{V} ; F(1,15)=7.13, p=.018]$. There was no validity effect for either voluntary attention $[$ valid $=3.48 \mu \mathrm{V}$, invalid $=3.16 \mu \mathrm{V} ; F(1,15)=1.35$, $p=.26$ ] or social attention [valid $=1.06 \mu \mathrm{V}$, invalid $=0.95 \mu \mathrm{V}$; $F(1,15)=0.17, p=.69]$.

Although Fig. 2a does show negative differences (for valid vs. invalid) in the social and voluntary conditions at around $300 \mathrm{~ms}$, these differences are much later than the $\mathrm{Nd}$ seen for reflexive attention, and the topographies during this time range reveal that this difference is actually driven by the $\mathrm{P} 3$ component (described in a subsequent section below, and shown in Fig. 4b). The timing and topographies suggest that the effects seen for reflexive attention are similar to an "Nd1" component, which has been suggested to be related to initial target selection (McDonald et al. 2001), whereas a later "Nd2" is thought to be related to further feature processing of the targets. Overall, the existence of multiple effects of reflexive attention before $200 \mathrm{~ms}$ suggests that early visual processing is affected robustly and automatically by reflexive attention. In contrast, the lack of any early effects of voluntary or social attention in the present study, combined with the previous literature showing that early effects can sometimes be found with these types of attention, suggest that the effects of voluntary and social attention on early visual processing may be much more dependent on stimulus and task parameters.
Mid-latency frontal effects (N2) Another attention effect that was not the focus of this study, but that was clearly evident in the waveforms, was observed over frontal scalp sites at a mid-latency range corresponding to a frontal N2 component. This effect appeared temporally between the early visual components and the later P3. The effects of voluntary attention first emerged at this stage of processing, suggesting a possible difference between voluntary orienting and the other two types of orienting. To test this anterior negativity, we conducted a $3 \times 2 \times 2 \times 2$ ANOVA with the factors attention type (voluntary, reflexive, and social), validity (valid and invalid), visual field (RVF and LVF), and electrode (the two electrodes circled in Fig. 3c). The latency window was determined individually for each type of attention by finding the maximum difference between valid and invalid trials within the range of 200-300 ms after target onset (social, 231-252 ms; voluntary, 235-255 ms; reflexive, 240-260 ms). Critically, this ANOVA revealed a significant interaction between attention type and validity $[F(2,14)=7.28, p<.005]$, as well as significant main effects of attention type [social $=1.53 \mu \mathrm{V}$, voluntary $=$ $2.39 \mu \mathrm{V}$, reflexive $=1.72 \mu \mathrm{V} ; F(2,14)=5.2, p<.05]$ and validity $[$ valid $=1.53 \mu \mathrm{V}$, invalid $=2.22 \mu \mathrm{V} ; F(1,15)=8.7$, $p<.01]$. However, unlike the early visual processing effect, which was unique to reflexive attention, processing at this stage was significantly affected by voluntary attention.

In separate ANOVAs for the individual pairs of attention types $(2 \times 2 \times 2 \times 2$ ANOVAs with the same factors as above, but with only two attention types each), there were significant interactions between validity and attention type for both the voluntary and reflexive attention comparison $[F(1,15)=8.98, p<.01]$ and the voluntary and social attention comparison $[F(1,15)=7.79, p<.05]$. In the analysis comparing reflexive and social attention, the interaction between attention type and validity was not significant $[F(1,15)=0.72, p=.41]$. Again, separate $2 \times 2 \times$ 2 ANOVAs with the factors validity, visual field, and electrode were conducted for all types of attention. For reflexive orienting, there was no significant main effect of validity [validity: $F(1,15)=0.15, p=.70$ ], whereas there was a highly robust validity effect for voluntary attention $[F(1,15)=13.41, p<.01]$. Although the interaction between social and voluntary attention suggested that this component might reflect a critical difference between voluntary and social attention, the separate analysis of the social attention condition revealed a near-significant trend toward a validity effect $[F(1,15)=4.02, p=.06]$. We discuss this effect further in the Discussion section.

Longer latency effects (P3) The amplitude of the P3 ERP component was tested with a $3 \times 2 \times 2 \times 2$ ANOVA with the factors attention type (voluntary, reflexive, and social), validity (valid and invalid), visual field (RVF 
a Target ERPs (Mid-latency frontal effect)

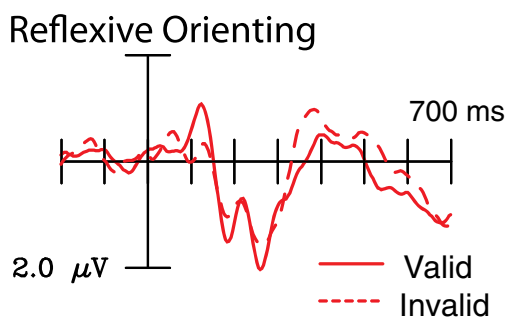

b

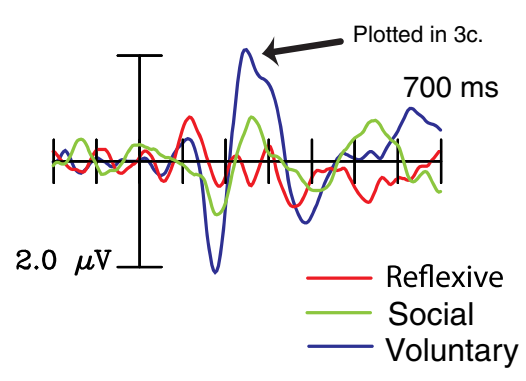

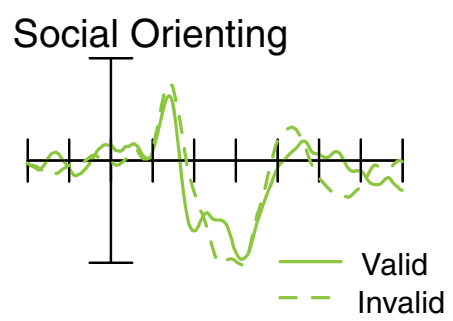

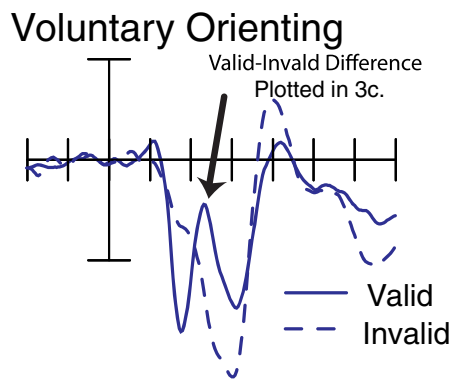

C Topographic map of voluntary attention difference
Fig. 3 Mid-latency frontal component. (a) Plots of valid and invalid waveforms for each type of attention, plotted at the electrode locations indicated in 3c. (b) Valid-minus-invalid difference waveforms for each attention type, at the electrodes indicated in 3c. Voluntary attention is

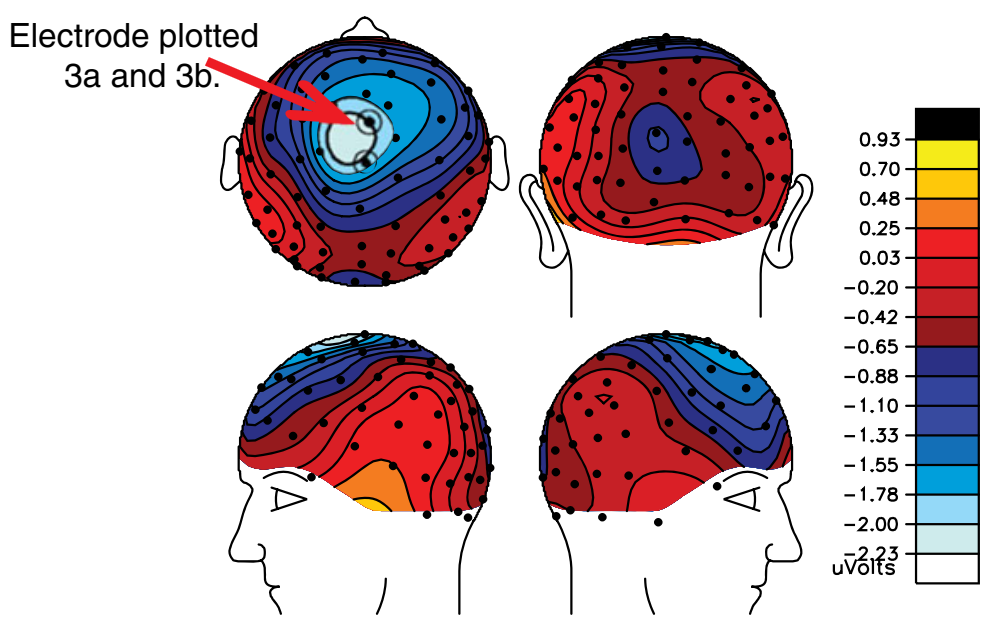

the only type showing significant validity differences at this mid-latency range. (c) Topographic map of the voluntary attention effect (difference wave plotted in $3 \mathrm{~b}$ ) for the time range of $235-255 \mathrm{~ms}$. The two electrodes that are circled were the ones used in the statistical analyses and LVF), and electrode (the two electrodes circles in Fig. 4b). The latency range was adjusted to ensure that the maximum amplitude of the $\mathrm{P} 3$ was included for each type of attention (voluntary, 277-297 ms; social, 275-295 ms; reflexive, $253-273 \mathrm{~ms}$ ). The analysis of the $\mathrm{P} 3$ revealed a significant validity effect [valid $=2.60 \mu \mathrm{V}$, invalid $=3.00 \mu \mathrm{V}$; $F(1,15)=8.07, p<.05]$ and, critically, a highly significant interaction between attention type and validity $[F(2,14)=$ $14.49, p<.001]$.

Separate ANOVAs for the pairs of levels of attention type were conducted to explore this interaction further. An ANOVA with voluntary and social attention revealed a significant main effect of validity $[F(1,15)=24.57, p<$ $.001]$, but no interaction between attention type and validity $[F(1,15)=0.36, p=.56]$, providing evidence that social and voluntary attention share a common effect on this stage of processing. There were significant interactions between validity and attention type, however, for both the comparison of reflexive and social orienting $[F(1,15)=14.88, p=$
.005] and the comparison of reflexive and voluntary orienting $[F(1,15)=15.94 ; p<.005]$. Therefore, the effect of reflexive attention at this late stage of processing is again distinctly different from the effects of social and voluntary orienting.

Separate $2 \times 2 \times 2$ ANOVAs with the factors validity, visual field, and electrode revealed significant main effects of validity for social attention $[F(1,15)=37.38, p<.001]$ and for voluntary attention $[F(1,15)=11.48, p<.005]$. For reflexive blocks, there was no main effect of validity $[F(1,15)=1.32, p=.27]$. However, it is noteworthy that the trend of the reflexive effect (valid greater than invalid) was in the direction opposite that for social and voluntary attention, providing further support for the distinction between social gaze orienting and reflexive attention. Thus, at late stages of processing, social attention shows effects that are qualitatively and quantitatively different from the effect of reflexive attention, but that are highly similar to the effects of voluntary orienting. 
a Target ERPs (Posterior P3)
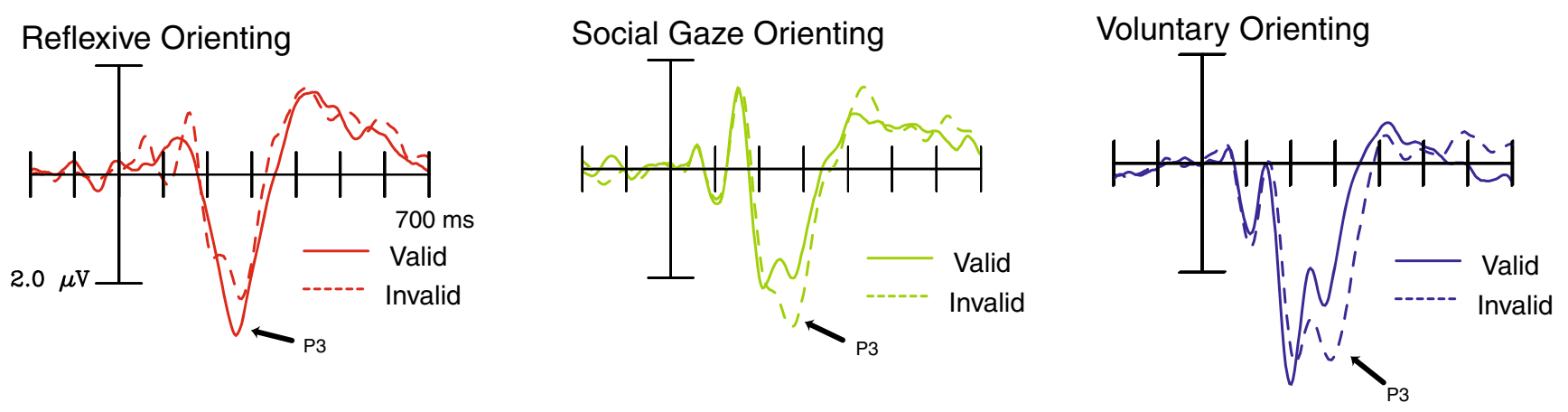

\section{b Topographic map of difference waves (valid - invalid) for the peak timing of the P3}

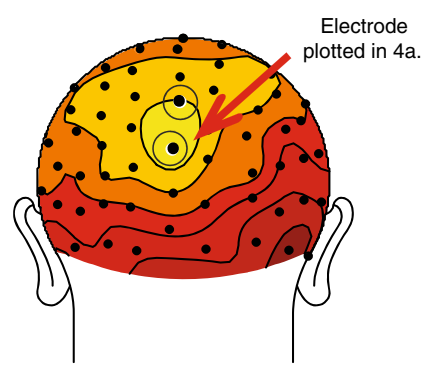

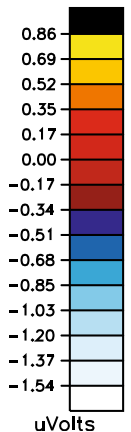

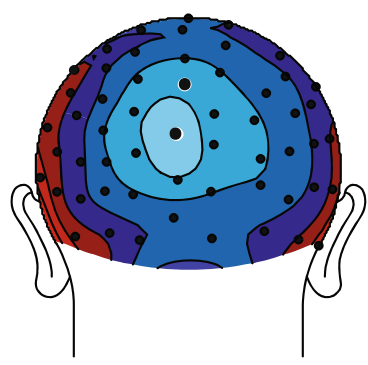

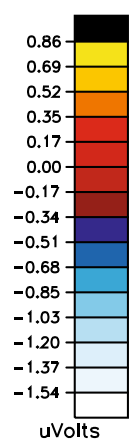

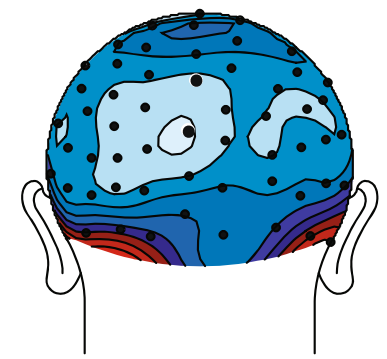

Fig. 4 Late posterior component (P3). (a) Plots of valid and invalid waveforms for each type of attention, plotted at the electrode locations indicated in 4 b. (b) Topographic map of the valid-minus-invalid difference (i.e., attention effect) for each attention type, plotted for the

\section{Discussion}

Although it is clear that social gaze cues affect behavioral responses to targets in a manner generally similar to voluntary and reflexive orienting, it has been unclear whether these types of orienting affect neural processing in the same way at multiple levels preceding overt responses. The present findings provide new evidence that social gaze orienting affects subsequent target processing in a manner that is qualitatively different from the effect of reflexive attention. Although both social gaze orienting and involuntary capture appear to be automatically triggered, and both show similar response facilitation in reaction times, the present data show that the underlying neural effects of these two types of attention differ at multiple stages of processing.

At an early stage of sensory processing (e.g., P1), only reflexive attention modulated target processing, suggesting that reflexive attention may work via mechanisms of stimulus enhancement (and/or suppression of irrelevant visual information), as was suggested by $\mathrm{Lu}$ and Dosher (1998, 2000). The use of neutral trials in a future study may further help to clarify whether the effect of reflexive peak time range for each type of attention (time ranges: reflexive, 253-273 ms; social, 275-295 ms; voluntary, 277-297 ms). The two electrodes that are circled were used in the statistical analyses

orienting on early visual processing is driven by enhanced processing of the valid target, or rather by suppressed processing of the invalid target. Previously, Luck, Hillyard, Mouloua, Woldorff, Clark and Hawkins (1994) used a neutral cue to conclude that the P1 attention effect for voluntary attention represents a suppression of processing at unattended locations, while the voluntary attention effect on the posterior $\mathrm{N} 1$ component reflects enhanced processing at the attended location.

At later stages (e.g., P3), the effects of social gaze orienting in the current study were qualitatively different from those of reflexive orienting. Specifically, gaze cuing produced a larger-amplitude P3 component for invalid than for valid targets, while the exact opposite pattern was found for targets following reflexive cues. It is interesting to note that social gaze cues were able to produce robust behavioral effects in the present study without any enhancement of early perceptual processing. Thus, these later decision-level effects, observed as modulations of the P3, may play a more important role in gaze orienting than has previously been thought, whereas reflexive attention may rely more heavily on perceptual facilitation. Thus, the present data provide new evidence dissociating social gaze orienting from reflexive capture and 
provide specificity with regards to the levels of processing where those differences emerge.

In contrast to the distinct effects of social versus reflexive orienting, voluntary and social gaze orienting produced very similar effects on target processing. Neither social nor voluntary orienting affected early sensory-level components in this task. Given that many previous studies have shown effects of voluntary attention at early stages of processing (e.g., Eimer, 1994b; Mangun \& Hillyard, 1991), we do not suggest that the present results should be interpreted as evidence that voluntary and social orienting cannot affect these levels of processing. Rather, the present results lend further support to the argument that the effects of voluntary attention on early visual processing are sensitive to task demands and perceptual load (Handy \& Mangun, 2000). Indeed, small and/or nonexistent effects of voluntary orienting on early sensory processing are not an uncommon finding when using a localization task (Eimer, 1994a; Prime \& Ward, 2006), such as the one used in this study. The present results suggest that social gaze orienting may be similar to voluntary attention in terms of the sensitivity of early attention effects to task parameters. Notably, the early effects of reflexive attention have been shown to be more automatic, in terms of not showing this sensitivity to task parameters (as reviewed by Hopfinger \& West, 2006). While the lack of early attention effects for the voluntary and social orienting conditions in the present study suggests a similarity at this level of processing, further within-subjects work will be required in order to better understand whether this similarity holds across multiple tasks and cognitive load conditions when early visual effects may be seen for these types of orienting.

The present pattern of results, with only reflexive attention affecting early perceptual processing, is in contrast to the conclusions of Prinzmetal and colleagues (Prinzmetal et al. 2010, 2005), who suggested that voluntary attention enhances the perceptual representation of the target (Prinzmetal et al. 2005), whereas reflexive attention works at a decision stage of processing (Prinzmetal et al. 2010). Task differences, however, are once again important to consider. Prinzmetal et al. (2005) compared voluntary and reflexive attention by manipulating the predictiveness of a peripheral onset. Such a design is useful for assessing how the addition of predictiveness modulates reflexive attention, but it is not ideal for separating and isolating the effects of voluntary attention, since the peripheral cue triggers reflexive orienting as well. In addition, if voluntary and reflexive attention interact, as previous research has suggested (Friesen et al. 2004; Hopfinger \& West, 2006), the use of a predictive peripheral cue may not simply add voluntary attention to reflexive attention, but may instead result in nonadditive interactions. Similarly, predictive social cues would potentially confound gaze orienting with voluntary attention. The present investigation was focused on the effects of each type of orienting on target processing; therefore, the target stimuli (which produced the ERPs analyzed here) were identical in each condition, and the cue stimuli were designed to isolate each type of attention. Whereas we were able to analyze the effects of each type of orienting, the use of physically different cues in the present study does prevent us from comparing ERP indices of attentional control processing.

At the level of the P3, social gaze orienting and voluntary attention produced similar effects, with largeramplitude activity for invalid than for valid targets. This finding provides additional evidence that the social gaze cuing effect on the P3 is robust, found across varying tasks. Furthermore, the similar effect found for voluntary attention suggests that this stage of processing may play a critical role in the behavioral consequences of both voluntary and social orienting. As described earlier, the P3 component has been linked to the perceived relevance of a stimulus and to context updating (Donchin, 1981; Duncan-Johnson \& Donchin, 1982). Furthermore, unexpected or "oddball" stimuli produce a larger P3 component than do more commonly occurring stimuli, possibly because the unexpected stimuli are potentially more important. With regard to social gaze orienting, targets occurring away from the gaze direction may thus be processed as odd, even though invalid and valid trials are equally probable with these cues. Perhaps, when experiencing social gaze cues in real-world situations, it is common that an interesting "target" would be present at the gazed-at location, while it is an infrequent occurrence for nothing to be present at the gazed-at location; therefore, the latter result may entail more effort in updating the current context.

A significant ERP difference between voluntary and social gaze orienting was observed in a frontal N2 component. Voluntary attention produced enhanced negativity for valid as compared to invalid targets, an effect that was not present for reflexive attention. Previous studies have reported a validity effect on a negative deflection of similar latency and have suggested that it may be a measure of attentional selection (e.g., Eimer, 1994a; Mangun \& Hillyard, 1991). While this validity effect is significant only for the voluntary condition in our study, this is not strong evidence for a qualitative difference in the effects of voluntary and social orienting, because there was a nearsignificant $(p=.06)$ trend toward an $\mathrm{N} 2$ validity effect in the social condition as well. Furthermore, the reaction time effect was much larger in the voluntary condition, suggesting that this difference between social and voluntary orienting may be a matter of the strength of the effect rather than an indication of a critical difference between these types of orienting. ${ }^{1}$ Future research focused on this

\footnotetext{
${ }^{1}$ The authors thank Clayton Hickey for this suggestion.
} 
component may reveal whether this difference represents a real distinction between these types of attention.

The present study provides new evidence for a distinction between the effects of social gaze orienting and reflexive orienting across multiple stages of processing. Although studies have shown similarities between these types of orienting, the present results argue that the similar effects on overt responses masked critical differences in what generated these effects. The present results also suggest that social and voluntary attention may share similar mechanisms of action, especially at the level of context updating and decision-level processing (as indexed by the $\mathrm{P} 3$ effect). If social and voluntary attention produce similar effects at multiple stages of processing, however, it may be necessary to reconsider previous data suggesting that voluntary and social gaze orienting are separable systems. A key piece of evidence has been the finding that counterpredictive social gaze cues produced speeded reaction times at both the gazed-at location and the predicted location (Friesen et al. 2004). However, this enhancement at more than one location is not necessarily evidence for separable systems, because it is known that voluntary attention can be spread across different-sized areas (LaBerge, 1983). Furthermore, debate continues as to whether voluntary attention can be split between two noncontiguous locations, or whether the area in-between is included in a broadened attended region (as reviewed by Cave \& Bichot, 1999). Therefore, even if social gaze cuing and voluntary orienting share some effects, it may be possible that multiple locations could show the effects simultaneously. Future research will be necessary to better assess the potential competition that may occur at multiple levels when social and voluntary orienting are concurrently engaged.

Finally, considering these similar effects of social gaze orienting and voluntary attention, it may be tempting to conclude that social gaze orienting co-opted part of a voluntary attention system. However, although voluntary attention has a much longer and richer history of research, it may instead be that the system that is typically measured with voluntary attention studies ties into the naturally developed social orienting system (Birmingham \& Kingstone, 2009). Regardless of the evolutionary history of these different types of orienting, the present results provide new data linking social gaze and voluntary orienting through similar effects on perceptual and postperceptual stages of target processing, and further distinguish these types of orienting from reflexive attentional capture.

\section{References}

Bayliss, A. P., \& Tipper, S. P. (2006). Predictive gaze cues and personality judgments: Should eye trust you? Psychological Science, 17, 514-520. doi:10.1111/j.1467-9280.2006.01737.x
Berger, A., Henik, A., \& Rafal, R. (2005). Competition between endogenous and exogenous orienting of visual attention. Journal of Experimental Psychology: General, 134, 207-221.

Birmingham, E., \& Kingstone, A. (2009). Human social attention: A new look at past, present, and future investigations. Annals of the New York Academy of Sciences, 1156, 118-140.

Briand, K. A. (1998). Feature integration and spatial attention: More evidence of a dissociation between endogenous and exogenous orienting. Journal of Experimental Psychology: Human Perception and Performance, 24, 1243-1256.

Cave, K. R., \& Bichot, N. P. (1999). Visuospatial attention: Beyond a spotlight model. Psychonomic Bulletin \& Review, 6, 204-223. doi: $10.3758 / \mathrm{BF} 03212327$

Cheal, M., \& Lyon, D. R. (1991). Central and peripheral precuing of forced-choice discrimination. Quarterly Journal of Experimental Psychology, 43A, 859-880. doi:10.1080/14640749108400960

Desimone, R., \& Duncan, J. (1995). Neural mechanisms of selective visual attention. Annual Review of Neuroscience, 18, 193-222. doi:10.1146/annurev.ne.18.030195.001205

Donchin, E. (1981). Surprise! .. Surprise? Psychophysiology, 18, 493513. doi:10.1111/j.1469-8986.1981.tb01815.x

Driver, J., Davis, G., Ricciardelli, P., Kidd, P., Maxwell, E., \& BaronCohen, S. (1999). Gaze perception triggers reflexive visuospatial orienting. Visual Cognition, 6, 509-540.

Duncan-Johnson, C., \& Donchin, E. (1982). The P300 component of the event-related potential as an index of information processing. Biological Psychology, 14, 1-52.

Eimer, M. (1994a). An ERP study on visual spatial priming with peripheral onsets. Psychophysiology, 31, 154-163. doi:10.1111/ j.1469-8986.1994.tb01035.x

Eimer, M. (1994b). "Sensory gating" as a mechanism for visuospatial orienting: Electrophysiological evidence from trial-by-trial cuing experiments. Perception \& Psychophysics, 55, 667-675. doi:10.3758/BF03211681

Fichtenholtz, H. M., Hopfinger, J. B., Graham, R., Detwiler, J. M., \& LaBar, K. S. (2007). Happy and fearful emotion in cues and targets modulates event-related potential indices of gaze-directed attentional orienting. Social Cognitive and Affective Neuroscience, 2, 323-333.

Fichtenholtz, H. M., Hopfinger, J. B., Graham, R., Detwiler, J. M., \& LaBar, K. S. (2009). Event-related potentials reveal temporal staging of dynamic emotional expression and gaze shift effects on attentional orienting. Social Neuroscience, 4, 317-331.

Friesen, C. K., \& Kingstone, A. (1998). The eyes have it! Reflexive orienting is triggered by nonpredictive gaze. Psychonomic Bulletin \& Review, 5, 490-495. doi:10.3758/BF03208827

Friesen, C. K., \& Kingstone, A. (2003). Abrupt onsets and gaze direction cues trigger independent reflexive attentional effects. Cognition, 87, B1-B10. doi:10.1016/S0010-0277(02)00181-6

Friesen, C. K., Ristic, J., \& Kingstone, A. (2004). Attentional effects of counterpredictive gaze and arrow cues. Journal of Experimental Psychology: Human Perception and Performance, 30, 319-329. doi:10.1037/0096-1523.30.2.319

Handy, T. C., Green, V., Klein, R., \& Mangun, G. R. (2001). Combined expectancies: ERPs reveal the early benefits of spatial attention that are obscured by reaction time measures. Journal of Experimental Psychology: Human Perception and Performance, 27, 303-317.

Handy, T. C., \& Mangun, G. R. (2000). Attention and spatial selection: Electrophysiological evidence for modulation by perceptual load. Perception \& Psychophysics, 62, 175-186.

Hietanen, J. K., Leppänen, J. M., Nummenmaa, L., \& Astikainen, P. (2008). Visuospatial attention shifts by gaze and arrow cues: An ERP study. Brain Research, 1215, 123-136. doi:10.1016/j. brainres.2008.03.091 
Hopfinger, J. B., \& Mangun, G. R. (1998). Reflexive attention modulates processing of visual stimuli in human extrastriate cortex. Psychological Science, 9, 441-447.

Hopfinger, J. B., \& Mangun, G. R. (2001). Tracking the influence of reflexive attention on sensory and cognitive processing. Cognitive, Affective, \& Behavioral Neuroscience, 1, 56-65.

Hopfinger, J. B., \& Ries, A. J. (2005). Automatic versus contingent mechanisms of sensory-driven neural biasing and reflexive attention. Journal of Cognitive Neuroscience, 17, 1341-1352.

Hopfinger, J. B., \& West, V. M. (2006). Interactions between endogenous and exogenous attention on cortical visual processing. Neurolmage, 31, 774-789.

Jonides, J. (1981). Voluntary versus automatic control over the mind's eye's movement. In J. Long \& A. Baddeley (Eds.), Attention and performance $I X$ (pp. 187-203). Hillsdale, NJ: Erlbaum.

Kennett, S., Eimer, M., Spence, C., \& Driver, J. (2001). Tactile-visual links in exogenous spatial attention under different postures: Convergent evidence from psychophysics and ERPs. Journal of Cognitive Neuroscience, 13, 462-478.

LaBerge, D. (1983). Spatial extent of attention to letters and words. Journal of Experimental Psychology: Human Perception and Performance, 9, 371-379.

Lu, Z.-L., \& Dosher, B. A. (1998). External noise distinguishes attention mechanisms. Vision Research, 38, 1183-1198. doi:10.1016/S0042-6989(97)00273-3

Lu, Z.-L., \& Dosher, B. A. (2000). Spatial attention: Different mechanisms for central and peripheral temporal precues? Journal of Experimental Psychology: Human Perception and Performance, 26, 1534-1548. doi:10.1037/0096-1523.26.5.1534

Luck, S. J., Hillyard, S. A., Mouloua, M., Woldorff, M. G., Clark, V. P., \& Hawkins, H. L. (1994). Effects of spatial cuing on luminance detectability: Psychophysical and electrophysiological evidence for early selection. Journal of Experimental Psychology: Human Perception and Performance, 20, 887-904.

Magnée, M. J. C. M., Kahn, R. S., Cahn, W., \& Kemner, C. (2011). More prolonged brain activity related to gaze cueing in schizophrenia. Clinical Neurophysiology, 122, 506-511.

Mangun, G. R., \& Hillyard, S. A. (1991). Modulations of sensoryevoked brain potentials indicate changes in perceptual processing during visual-spatial priming. Journal of Experimental Psychology: Human Perception and Performance, 17, 1057-1074.

McDonald, J. J., Teder-Sälejärvi, W. A., DiRusso, F., \& Hillyard, S. A. (2003). Neural substrates of perceptual enhancement by crossmodal spatial attention. Journal of Cognitive Neuroscience, 15, $10-19$.

McDonald, J. J., Teder-Sälejärvi, W. A., Heraldez, D., \& Hillyard, S. A. (2001). Electrophysiological evidence for the "missing link" in crossmodal attention. Canadian Journal of Experimental Psychology, 55, 141-149.

McDonald, J. J., \& Ward, L. M. (2000). Involuntary listening aids seeing: Evidence from human electrophysiology. Psychological Science, 11, 167-171.

Müller, H. J., \& Rabbitt, P. M. (1989). Reflexive and voluntary orienting of visual attention: Time course of activation and resistance to interruption. Journal of Experimental Psychology: Human Perception and Performance, 15, 315-330. doi:10.1037/ 0096-1523.15.2.315

Posner, M. I. (1980). Orienting of attention. Quarterly Journal of Experimental Psychology, 32, 3-25. doi:10.1080/ 00335558008248231

Posner, M. I., \& Cohen, Y. (1984). Components of visual orienting. In H. Bouma \& D. G. Bouwhuis (Eds.), Attention and performance X: Control of language processes (pp. 531-556). Hillsdale, NJ: Erlbaum.

Prime, D. J., \& Ward, L. M. (2006). Cortical expressions of inhibition of return. Brain Research, 1072, 161-174.

Prinzmetal, W., Ha, R., \& Khani, A. (2010). The mechanisms of involuntary attention. Journal of Experimental Psychology: Human Perception and Performance, 36, 255-267.

Prinzmetal, W., McCool, C., \& Park, S. (2005). Attention: Reaction time and accuracy reveal different mechanisms. Journal of Experimental Psychology: General, 134, 73-92.

Schuller, A.-M., \& Rossion, B. (2001). Spatial attention triggered by eye gaze increases and speeds up early visual activity. Neuroreport, 12, 2381-2386.

Schuller, A.-M., \& Rossion, B. (2004). Perception of static eye gaze direction facilitates subsequent early visual processing. Clinical Neurophysiology, 115, 1161-1168.

Schuller, A.-M., \& Rossion, B. (2005). Spatial attention triggered by eye gaze enhances and speeds up visual processing in upper and lower fields beyond early striate visual processing. Clinical Neurophysiology, 116, 2565-2576.

Störmer, V. S., McDonald, J. J., \& Hillyard, S. A. (2009). Cross-modal cueing of attention alters appearance and early cortical processing of visual stimuli. Proceedings of the National Academy of Sciences, 106, 22456-22461.

Tipper, C. M., Handy, T. C., Giesbrecht, B., \& Kingstone, A. (2008). Brain responses to biological relevance. Journal of Cognitive Neuroscience, 20, 879-891.

Vogel, E. K., \& Luck, S. J. (2000). The visual N1 component as an index of a discrimination process. Psychophysiology, 37, 190 203. doi: $10.1017 / \mathrm{S} 0048577200981265$

Woldorff, M. G. (1993). Distortion of ERP averages due to overlap from temporally adjacent ERPs: Analysis and correction. Psychophysiology, 30, 98-119. 